\title{
6 The problem of incommensurabilities
}

The third issue that the argument from the Basic Representation Theorem raised, besides the problem of intertheoretic comparisons and that of probabilities, was the problem of incommensurabilities. By assuming Completeness, the arguments so far rule out any incommensurabilities, and thus suffer from two major restrictions. As indicated in Chapter 3, many plausible axiologies allow for incommensurabilities at the level of first-order value, and are thus themselves incomplete. And as substantiated in Chapter 4, even if there's no fundamental problem with intertheoretic comparisons or the idea of EVM, plausibly, some axiologies aren't fully commensurable with each other, and thus induce an incomplete m-value relation. A theorem presupposing Completeness can't allow for such incommensurabilities. So it's time to introduce a representation theorem that doesn't presuppose this axiom. I'll do that in the present chapter (Section 6.1). The emerging theorem will be the most encompassing result of this book.

Having dropped Completeness, we'll be in a better position to assess whether representation theorems can ground EVM as a general theory of moral-rather than just axiological-uncertainty. So to round off my exploration of the axiomatic approach, I'll identify the most promising strategies to extend my arguments beyond axiology, and the most serious difficulties these strategies face (Section 6.2). I'll end the chapter with a conclusion, summarising the main positive and negative upshots of the book, and indicating possible directions for further research (Section 6.3).

\subsection{Axiomatising incomplete $\mathrm{m}$-value relations}

To explicate and defend a form of EVM covering incommensurabilities, I'll again first state a relevant theorem in terms of mathematical functions, and then interpret it in a philosophically significant manner. And I'll again start with a theorem that assumes probabilities as given, before extending it to allow for an explication of them.

\section{The Expected Value Theorem for Incompleteness}

Consider the relation $\succeq_{m}$ on $\mathcal{Q}$. To allow for incompleteness, we'll need a slightly different set of axioms. Let $\boldsymbol{a}_{(i, x)}$ be the prospect that certainly leads to $(i, x)$ : $\boldsymbol{a}_{(i, x)}(i, x)=1$. For a reflexive binary relation $\geq$ on $\mathcal{Q}$, define 
Transitivity $_{\mathcal{Q}}$ : for all $\boldsymbol{a}, \boldsymbol{b}$ and $\boldsymbol{c}$ in $\mathcal{Q}$, if $\boldsymbol{a} \geq \boldsymbol{b}$ and $\boldsymbol{b} \geq \boldsymbol{c}$, then $\boldsymbol{a} \geq \boldsymbol{c}$;

Mixture-Independence $_{\mathcal{Q}}$ : for all $\boldsymbol{a}$ and $\boldsymbol{b}$ in $\mathcal{Q}, \boldsymbol{a} \geq \boldsymbol{b}$ if and only if $p \boldsymbol{a}+(1-p) \boldsymbol{c} \geq$ $p \boldsymbol{b}+(1-p) \boldsymbol{c}$ for all $p \in] 0,1[$ and all $\boldsymbol{c}$ in $\mathcal{Q}$;

Sequence-Continuity $_{\mathcal{Q}}$ : if $\left\{\boldsymbol{a}_{n}\right\}$ and $\left\{\boldsymbol{b}_{n}\right\}$ are convergent sequences such that $\boldsymbol{a}_{n} \geq \boldsymbol{b}_{n}$ for all $n$, then $\lim \left(\boldsymbol{a}_{n}\right) \geq \lim \left(\boldsymbol{b}_{n}\right)$;

Existence of Best and Worst ${ }_{\mathcal{Q}}$ : there are $(\overline{i, x})$ and $(i, x)$ in $(I \times X)$ such that for all $(i, x)$ in $(I \times X), \boldsymbol{a}_{(\overline{i, x})} \geq \boldsymbol{a}_{(i, x)}$, and $\boldsymbol{a}_{(i, x)} \geq \boldsymbol{a}_{(i, x)}$; and

Non-Triviality $\mathcal{Q}_{\mathcal{Q}}: \boldsymbol{a}_{(\overline{i, x})}>\boldsymbol{a}_{(i, x)}$ (i. e., not $\left.\boldsymbol{a}_{(\overline{i, x})} \geq \boldsymbol{a}_{(\overline{i, x})}\right)$.

If a reflexive relation $\geq$ on $\mathcal{Q}$ satisfies these axioms, I'll say it is ' $N$-conformable' (since my results are based on Nau 2006). Note that Existence of Best and Worst implies that $\succeq$ can't be totally incomplete. But apart from this, these conditions don't assume that $\succeq$ is complete. Now instead of defining a uniqueness criterion for our utility functions-which is somewhat more complicated if we're dealing with incomplete relations ${ }^{129}$-we'll simply introduce a normalisation for these functions, and then focus on normalised functions only. So define a normalised set of utility functions

$$
\begin{aligned}
U^{\star}=\{u: I \times X \rightarrow \mathbb{R} \mid u(\overline{i, x}) & =0 ; 0 \leq u(i, x) \leq 1 \forall(i, x) \in(I, X) ; \\
u(\overline{i, x}) & =1\} .
\end{aligned}
$$

Call a collection of preferences $\left\{\boldsymbol{a}_{n} \geq \boldsymbol{b}_{n}\right\}$ a basis for $\geq$ under an axiom system if every preference $\boldsymbol{a} \geq \boldsymbol{b}$ can be deduced from $\left\{\boldsymbol{a}_{n} \geq \boldsymbol{b}_{n}\right\}$ by application of these axioms. Finally, for simplicity, for some function $u: I \times X \rightarrow \mathbb{R}$, define

$$
\mathbf{U}_{u}(\boldsymbol{a})=\sum_{i \in I, x \in X} \boldsymbol{a}(i, x) u(i, x)
$$

It can be shown ${ }^{130}$ that if $\succeq_{m}$ is $\mathrm{N}$-conformable, there exists a nonempty closed convex set $U \subset U^{\star}$ of functions, such that for all $\boldsymbol{a}$ and $\boldsymbol{b}$ in $\mathcal{Q}$,

$$
\boldsymbol{a} \geq_{m} \boldsymbol{b} \text { iff } \sum_{i \in I, x \in X} \boldsymbol{a}(i, x) u(i, x) \geq \sum_{i \in I, x \in X} \boldsymbol{b}(i, x) u(i, x) \quad \forall u \in U
$$

In particular, if $\left\{\boldsymbol{a}_{n} \geq_{m} \boldsymbol{b}_{n}\right\}$ is a basis for $\succeq_{m}$ under these axioms, then $U$ is the set of $u$ in $U^{\star}$ satisfying $\left\{\mathbf{U}_{u}\left(\boldsymbol{a}_{n}\right) \geq \mathbf{U}_{u}\left(\boldsymbol{b}_{n}\right)\right\}$. (6.3) is a representation in terms of a set of utility functions. This straightforwardly allows for incompleteness in $\succeq_{m}$ : neither

129 See Nau (2006) for a fully spelt out uniqueness criterion.

130 See the appendix (Section A.3; 'The Representation Theorem for Incompleteness') for a proof. 
$\boldsymbol{a} \geq_{m} \boldsymbol{b}$ nor $\boldsymbol{b} \geq_{m} \boldsymbol{a}$ are true if there are $u$ and $v$ in $W$ with $\mathbf{U}_{u}(\boldsymbol{a})>\mathbf{U}_{u}(\boldsymbol{b})$ and $\mathbf{U}_{v}(\boldsymbol{b})>\mathbf{U}_{v}(\boldsymbol{a})$.

We again need to ensure that the relevant utility functions $u$ in $U^{\star}$ are appropriately related to our axiologies, by assuming that our axiologies satisfy certain conditions, and that $\succeq_{m}$ is related to them via a Pareto condition. For a reflexive binary relation $\succeq$ on $\mathcal{O}$, define

Transitivity $\mathcal{O}_{\mathcal{O}}$ for all $a, b$ and $c$ in $\mathcal{O}$, if $a \geq b$ and $b \geq c$, then $a \geq c$;

Mixture-Independence $\mathcal{O}_{\mathcal{O}}$ : for all $a$ and $b$ in $\mathcal{O}, a \geq b$ if and only if $p a+(1-p) c \geq$ $p b+(1-p) c$ for all $p \in] 0,1[$ and all $c$ in $\mathcal{O}$; and

Sequence-Continuity $\mathbf{O}_{\mathcal{O}}$ : if $\left\{a_{n}\right\}$ and $\left\{b_{n}\right\}$ are convergent sequences such that $a_{n} \geq b_{n}$ for all $n$, then $\lim \left(a_{n}\right) \geq \lim \left(b_{n}\right)$.

If a reflexive relation $\succeq$ on $\mathcal{O}$ satisfies these conditions, I'll say it's $N^{*}$-conformable. For a set of binary relations $\left\{\geq_{i} \mid i \in I\right\}$ on $\mathcal{O}$ and a binary relation $\succeq$ on $\mathcal{Q}$, define the

Strong Pareto Condition: For any probability distribution $P$ on $I$, and for all $\boldsymbol{a}$ and $\boldsymbol{b}$ in $\mathcal{Q}^{P}$, if $H_{i}(\boldsymbol{a}) \sim_{i} H_{i}(\boldsymbol{b})$ for all $i$ in $I$ with $P(i)>0$, then $\boldsymbol{a} \sim \boldsymbol{b}$; if $H_{i}(\boldsymbol{a}) \geq_{i}$ $H_{i}(\boldsymbol{b})$ for all $i$ in $I$ with $P(i)>0$ and $H_{j}(\boldsymbol{a})>_{j} H_{j}(\boldsymbol{b})$ for some $j$ in $I$ with $P(j)>0$, then $\boldsymbol{a}>\boldsymbol{b}$; and if for some $j$ in $I$ with $P(j)>0, H_{i}(\boldsymbol{a}) \sim_{i} H_{i}(\boldsymbol{b})$ for all $i$ in $I$ with $i \neq j$ and $P(i)>0$, then $\boldsymbol{a} \geq \boldsymbol{b}$ only if $H_{j}(\boldsymbol{a}) \geq_{j} H_{j}(\boldsymbol{b})$.

The third clause of this condition guarantees that the value functions in our representation will not represent sharpenings of our axiologies.

Now the following theorem holds:

Representation Theorem for Incompleteness: Suppose that all our $\geq_{i}$ are $N^{*}$-conformable. If $\geq_{m}$ is $N$-conformable and satisfies the Strong Pareto Condition with respect to our $\geq_{i}$, there's a nonempty closed convex set $U \subset U^{*}$ of functions, such that for all $\boldsymbol{a}$ and $\boldsymbol{b}$ in $\mathcal{Q}$, all $a$ and $b$ in $\mathcal{O}$ and all $i$ in $I$,

$$
\begin{gathered}
\boldsymbol{a} \geq_{m} \boldsymbol{b} \text { iff } \sum_{i \in I, x \in X} \boldsymbol{a}(i, x) u(i, x) \geq \sum_{i \in I, x \in X} \boldsymbol{b}(i, x) u(i, x) \quad \forall u \in U, \quad \text { and } \\
a \geq_{i} b \text { iff } \sum_{x \in X} a(x) u(i, x) \geq \sum_{x \in X} b(x) u(i, x) \quad \forall u \in U .
\end{gathered}
$$

In particular, if $\left\{\boldsymbol{a}_{n} \geq_{m} \boldsymbol{b}_{n}\right\}$ is a basis for $\geq_{m}$ under these axioms, then $U$ is the set of $u$ in $U^{*}$ satisfying $\left\{\mathbf{U}_{u}\left(\boldsymbol{a}_{n}\right) \geq \mathbf{U}_{u}\left(\boldsymbol{b}_{n}\right)\right\} .131$

131 See the appendix (Section A.3) for a proof. 
To turn this into a non-mathematical theorem, we again need prospect-explications of intra- and intertheoretic comparisons. These will have to be slightly different from the ones we've got so far. If we want to represent an axiology on which outcomes are less than fully commensurable, we can't interpret it as implying determinate value-difference ratios of the form 'the value-difference between $x$ and $y$ is $n$ times as great as the value-difference between $z$ and $t$ '. Similarly, if we want to represent two axiologies that aren't fully commensurable, we can't interpret them as implying determinate intertheoretic value-difference ratios of the form 'the value-difference between $x$ and $y$, according to $T_{i}$, is $n$ times as great as the value-difference between $z$ and $t$, according to $T_{j}$ '. Instead, as far as intratheoretic comparisons are concerned we're interested in rough cardinal intratheoretic value comparisons, or facts of the form

(U) according to $T_{i}$, the value-difference between $x$ and $y$ is at least (or at most) $n$ times as great as the value-difference between $z$ and $t$.

As far as intertheoretic comparisons are concerned, we're interested in rough cardinal intertheoretic value comparisons, or facts of the form

(V) the value-difference between $x$ and $y$, according to $T_{i}$, is at least (or at most) $n$ times as great as the value-difference between $z$ and $t$, according to $T_{j}$.

Again, our theorem guarantees that we can explicate level-comparisons too. That is, we can actually explicate rough crosscutting cardinal intertheoretic value comparisons, or facts of the form

(W) the difference between the value of $x$, according to $T_{i}$, and the value of $y$, according to $T_{j}$, is at least (or at most) $n$ times as great as the difference between the value of $z$, according to $T_{h}$, and the value of $t$, according to $T_{k}$.

To state our explications, let $U$ be a nonempty closed convex set of utility functions $u: I \times X \rightarrow \mathbb{R}$. Suppose that for some axiology $T_{i}$, and for all $a$ and $b$ in $\mathcal{O}$,

$$
a \geq_{i} b \quad \text { iff } \quad \sum_{x \in X} a(x) u(i, x) \geq \sum_{x \in X} b(x) u(i, x) \quad \forall u \in U
$$

I'll then say that $U$ represents $T_{i}$ ordinally. And I'll use equivalent, self-explanatory definitions for the claims that some nonempty closed convex set $U$ of utility functions represents the m-value relation $\succeq_{m}$ ordinally, or (below) that some pair $(U, P)$ represents your $\succeq_{m}$ and $\tilde{\succeq}_{m}$ ordinally. Now suppose that for some nonempty closed convex set $U$ of real-valued functions on $I \times X$, and some axiology $T_{i}$, the rough cardinal intratheoretic comparisons between outcomes, according to $T_{i}$, are true if and only if they are true for all functions $u(i, \cdot)$ with $u$ in $U$-that is, 
for all $x, y, z, t$ in $X$ (with $a_{x} \geq_{i} a_{y}$ and $a_{z} \geq_{i} a_{t}$ ), and $n \in \mathbb{R}$, the value-difference between $x$ and $y$ is at least $n$ times as great as the value-difference between $z$ and $t$, according to $T_{i}$, if and only if $(u(i, x)-u(i, y)) /(u(i, z)-u(i, t)) \geq n$ for all $u$ in $U$. I'll then say that $U$ represents $T_{i}$ cardinally. The prospect-explication of rough intratheoretic comparisons says that if there's a nonempty closed convex set $U \subset U^{\star}$ of utility functions, which represents an axiology ordinally, then it also represents it cardinally. Similarly, suppose that for some nonempty closed convex set $U$ of real-valued functions on $I \times X$, the rough crosscutting cardinal intertheoretic comparisons are true, according to our theories, if and only if they are true for all functions $u$ in $U$-that is, for all $x, y, z, t$ in $X$, all $i, j, h, k$ in $I$ (with $\boldsymbol{a}_{(i, x)} \geq_{m} \boldsymbol{a}_{(j, y)}$ and $\left.\boldsymbol{a}_{(h, z)} \geq_{m} \boldsymbol{a}_{(k, t)}\right)$ and $n \in \mathbb{R}$, the difference between the value of $x$, according to $T_{i}$, and the value of $y$, according to $T_{j}$, is at least $n$ times as great as the difference between the value of $z$, according to $T_{h}$, and the value of $t$, according to $T_{k}$, if and only if $(u(i, x)-u(j, y)) /(u(h, z)-u(k, t)) \geq n$ for all $u$ in $U$. I'll then say that $U$ jointly represents all axiologies cardinally. The prospect-explication of rough intertheoretic comparisons says that if there's a nonempty closed convex set $U \subset U^{\star}$ of theory-dependent utility functions, which represents the m-value relation ordinally, and represents each axiology cardinally, then it jointly represents all axiologies cardinally. If that's so, we can assume, say, that our theories are represented by the set of value functions $\mathcal{G}=\{G: I \times X \rightarrow \mathbb{R} \mid G=u, u \in U\}$, and accordingly, that each theory $T_{i}$ is represented by the set of value functions $\mathcal{G}_{i}=\left\{G_{i}: X \rightarrow \mathbb{R} \mid G_{i}=G(i, \cdot), G \in \mathcal{G}\right\}$.

Given these explications, the following theorem holds:

Expected Value Theorem for Incompleteness: Suppose that all our $\succeq_{i}$ are $N^{*}$-conformable. If $\succeq_{m}$ is $N$-conformable and satisfies the Strong Pareto Condition with respect to our $\succeq_{i}$, then for all $\boldsymbol{a}$ and $\boldsymbol{b}$ in $\mathcal{Q}$,

$$
\boldsymbol{a} \geq_{m} \boldsymbol{b} \text { iff } \sum_{i \in I, x \in X} \boldsymbol{a}(i, x) G_{i}(x) \geq \sum_{i \in I, x \in X} \boldsymbol{b}(i, x) G_{i}(x) \quad \forall G \in \mathcal{G} .^{132}
$$

Existence of Best and Worst implies that our axiologies can't be fully incomparable. Sequence-Continuity implies they can't compare in a lexical way. But I take it that such theories are extreme and comparatively very implausible. So this is a much less severe restriction than that imposed by Completeness. What's important is that (6.7) allows for both (non-radical) intra- and intertheoretic incomparability. If a theory $T_{i}$ features some intratheoretic incomparability, then some two functions in $\mathcal{G}_{i}$ aren't positive affine transformations of each other. If no axiology

132 See the appendix (Section A.3) for the derivation of this theorem from the Representation Theorem for Incompleteness and the prospect-explications. 
under consideration features intratheoretic incomparability, then for all $i$ in $I$, all functions in $\mathcal{G}_{i}$ are positive affine transformations of each other. There may then still be intertheoretic incomparability. In that case, for at least some theory $T_{i}$, not all functions in $\mathcal{G}_{i}$ are the same. Some of them are nontrivial positive affine transformations of each other.

\section{The Expected Value Theorem for Incompleteness and Probabilities}

Let's now make first steps towards axiomatising incomplete $\mathrm{m}$-value relations without given probabilities. To that end, we'll have to consider the relation $\widetilde{\succeq}_{m}$ on $\mathcal{K}$ again. And we must define our new axioms for this relation. Let $a_{x}$ be the prospect that for all $i$ in $I$ certainly leads to $x: a_{x}(i, x)=1$ for all $i$. For a reflexive binary relation $\tilde{z}$ on $\mathcal{K}$, define

Transitivity $_{\mathcal{K}}$ : for all $a, b$ and $c$ in $\mathcal{K}$, if $a \tilde{\geq} b$ and $b \tilde{\geq} c$, then $a \tilde{\geq} c$;

Mixture-Independence $\mathcal{K}_{\mathcal{K}}$ : for all $a$ and 6 in $\mathcal{K}, a \tilde{\leq} 6$ if and only if $p a+(1-$ $p) c \tilde{\leq} p b+(1-p) c$ for all $p \in] 0,1[$ and all $c$ in $\mathcal{K}$;

Sequence-Continuity $\mathcal{K}_{\mathcal{K}}$ : if $\left\{a_{n}\right\}$ and $\left\{b_{n}\right\}$ are convergent sequences such that $a_{n} \tilde{z} b_{n}$ for all $n$, then $\lim \left(a_{n}\right) \tilde{z} \lim \left(b_{n}\right)$;

Existence of Best and Worst ${ }_{\mathcal{K}}$ : there are $\bar{x}$ and $\underline{x}$ in $X$ such that for all $x$ in $X$, $a_{\bar{x}} \tilde{z} a_{x}$, and $a_{x} \tilde{z} a_{\underline{x}}$; and

Non-Triviality $\mathcal{K}_{\mathcal{K}}: a_{\bar{x}} \widetilde{\succ} a_{\underline{x}}$ (i. e., not $a_{\underline{x}} \tilde{z} a_{\bar{x}}$ ).

If a reflexive relation $\tilde{\underline{z}}$ on $\mathcal{K}$ satisfies these axioms, I'll again say it's $\mathrm{N}$-conformable. The incompleteness of $\widetilde{\Xi}_{m}$ on $\mathcal{K}$ raises a further complexity, noted on page 95. There are two reasons for why the $\mathrm{m}$-value relation relative to your credences may be incomplete. You may have credence in theories that give rise to incommensurability; or you may have fuzzy credences. As mentioned, for simplicity I'll ignore the latter case, and assume that the incompleteness of your m-value judgments is entirely due to incommensurability in your values.

To model this, say that a probability distribution $P$ on $I$ is positive if $P(i)>0$ for all $i$ in $I$. For binary relations $\geq$ on $\mathcal{Q}$ and $\tilde{z}$ on $\mathcal{K}$, define the

Reduction Axiom: There's a positive probability distribution $P$ on $I$ such that for all $\boldsymbol{a}$ and $\boldsymbol{b}$ in $\mathcal{Q}^{P}, \boldsymbol{a} \geq \boldsymbol{b}$ if and only if $L(\boldsymbol{a}) \tilde{\Sigma} L(\boldsymbol{b})$.

If your preferences on $\mathcal{K}$ satisfy the Reduction Axiom, they're exactly equal to your preferences on prospects conditional on the probability distribution $P$. I'm not aware that this axiom has been used in the literature for reducing incompleteness in preferences to incompleteness in values. Similar axioms have been used, 
but the ones I know of all depend on the assumption of state-independent preferences. ${ }^{133}$ It's undoubtedly very strong. It not only rules out fuzzy credences, but also basically gives us the required probabilities. But at least as a first step towards bringing together axiomatisations without Completeness and axiomatisations for state-dependent utilities, it's interesting to see what this axiom implies.

Say that $\succeq_{i}$ is strictly non-uniform if there are $\bar{x}_{i}, \tilde{x}_{i}, x_{i}$ and $\underline{x}_{i}$ in $X$ such that $a_{\bar{x}}>_{i} a_{\tilde{x}}, a_{\tilde{x}}>_{i} a_{\tilde{x}}$ and $a_{\underline{x}}>_{i} a_{\underline{x}}$. Given this definition, we can state the

Representation Theorem for Incompleteness and Probabilities: Suppose that all your $\succeq_{i}$ are $N^{*}$-conformable and strictly non-uniform. If your $\succeq_{m}$ and your $\tilde{\succeq}_{m}$ are $N$ conformable and jointly satisfy the Reduction Axiom for some positive probability distribution $P$ on I, and if your $\succeq_{m}$ satisfies the Strong Pareto Condition with respect to your $\succeq_{i}$, there's a nonempty closed convex set $U \subset U^{*}$ of functions such that for all $a$ and $b$ in $\mathcal{K}$, all $\boldsymbol{a}$ and $\boldsymbol{b}$ in $\mathcal{Q}$, all $a$ and $b$ in $\mathcal{O}$ and all $i$ in $I$,

$$
\begin{gathered}
a \Sigma_{m} b \text { iff } \sum_{i \in I, x \in X} a(i, x) P(i) u(i, x) \geq \sum_{i \in I, x \in X} b(i, x) P(i) u(i, x) \quad \forall u \in U, \\
\boldsymbol{a} \geq_{m} \boldsymbol{b} \text { iff } \sum_{i \in I, x \in X} \boldsymbol{a}(i, x) u(i, x) \geq \sum_{i \in I, x \in X} \boldsymbol{b}(i, x) u(i, x) \quad \forall u \in U, \quad \text { and } \\
a \geq_{i} b \text { iff } \sum_{x \in X} a(x) u(i, x) \geq \sum_{x \in X} b(x) u(i, x) \quad \forall u \in U .
\end{gathered}
$$

In particular, if $\left\{\boldsymbol{a}_{n} \geq_{m} \boldsymbol{b}_{n}\right\}$ is a basis for $\geq_{m}$ under these axioms, then $U$ is the set of $u \in U^{*}$ satisfying $\left\{\mathbf{U}_{u}\left(\boldsymbol{a}_{n}\right) \geq \mathbf{U}_{u}\left(\boldsymbol{b}_{n}\right)\right\}$. And there's no other probability distribution $Q \neq P$ for which this is true. ${ }^{134}$

To turn this into a philosophically significant result, we can expand the judgmentexplication from Chapter 5. Suppose that for some probability distribution $P$ on $I$ and some nonempty closed convex set $U$ of real-valued functions on $I \times X$, the rough crosscutting cardinal intertheoretic comparisons are true, according to your theories, if and only if they are true for all functions $u$ in $U$, and that your credence in any axiology $T_{i}$ is $P(i)$-that is, for all $x, y, z, t$ in $X$, all $i, j, h, k$ in $I$ (with $\boldsymbol{a}_{(i, x)} \succeq_{m} \boldsymbol{a}_{(j, y)}$ and $\left.\boldsymbol{a}_{(h, z)} \succeq_{m} \boldsymbol{a}_{(k, t)}\right)$ and $n \in \mathbb{R}$, the difference between the value of $x$, according to $T_{i}$, and the value of $y$, according to $T_{j}$, is at least $n$ times as great as the difference between the value of $z$, according to $T_{h}$, and the value of $t$, according to $T_{k}$, if and only if $(u(i, x)-u(j, y)) /(u(h, z)-u(k, t)) \geq n$ for all $u$ in $U$, with $p_{i}=P(i), p_{j}=P(j), p_{h}=P(h)$ and $p_{k}=P(k)$. I'll then say that $(U, P)$ represents your intertheoretic comparisons cardinally and your axiological probabilities

133 See e. g. the 'Reduction Axiom' in Ok et al. (2012).

134 See the appendix (Section A.3) for a proof. 
quantitatively. The judgment-explication of your rough intertheoretic comparisons and axiological probabilities says that if there's a unique pair $\left(U \subset U^{\star}, P\right)$, which represents your $\succeq_{m}$ and $\check{\succeq}_{m}$ ordinally, and if $U$ represents each of your axiologies cardinally, then $(U, P)$ represents your intertheoretic comparisons cardinally and your axiological probabilities quantitatively. Hence if that's so, we can assume that $P(i)$ represents your credence $p_{i}$ in the theory represented by the functions $\mathcal{G}_{i}=\left\{G_{i}: X \rightarrow \mathbb{R} \mid G_{i}=G(i, \cdot), G \in \mathcal{G}\right\}$.

Given this explication, the following theorem holds:

Expected Value Theorem for Incompleteness and Probabilities: Suppose that all your $\geq_{i}$ are $N^{*}$-conformable and strictly non-uniform. If your $\succeq_{m}$ and your $\tilde{\succeq}_{m}$ are $N$ conformable and jointly satisfy the Reduction Axiom for some positive probability distribution $P$ on I, and if your $\succeq_{m}$ satisfies the Strong Pareto Condition with respect to your $\succeq_{i}$, then for all $a$ and $b$ in $\mathcal{K}$,

$$
a \tilde{\succeq}_{m} b \quad \text { iff } \sum_{i \in I, x \in X} a(i, x) p_{i} G_{i}(x) \geq \sum_{i \in I, x \in X} b(i, x) p_{i} G_{i}(x) \quad \forall G \in \mathcal{G G}^{135}
$$

This is the most encompassing theorem of this book. Again, it doesn't allow us to represent fully incomparable theories, or theories that compare in a lexical way. And it doesn't allow for fuzzy credences. Moreover, that your $\succeq_{m}$ and $\widetilde{\succeq}_{m}$ should satisfy the Reduction Axiom is only plausible if you assign nonzero credence to all axiologies under consideration. So to ground a normative argument on this theorem, we'd have to restrict the set of axiologies under consideration accordingly. But these seem relatively minor restrictions. Given these restrictions, and in light of our account of intertheoretic comparisons, the remaining axioms seem plausible. So we've now overcome all the major problems that affected the argument from the Basic Representation Theorem. We have an axiomatic foundation for EVM that doesn't suffer from any fundamental problem of intertheoretic comparisons, doesn't take axiological probabilities as primitives, and doesn't rule out either intra- or intertheoretic incommensurabilities.

\subsection{General moral uncertainty}

We're now in a position to explore a final question the axiomatic approach raises. Can the argument I've given ground a general theory of normative rather than

135 See the appendix (Section A.3) for the derivation of this theorem from the Representation Theorem for Incompleteness and Probabilities and the judgment-explications. 
merely axiological uncertainty? That's the question of this section. Or more precisely, for simplicity, I'll ask whether our argument can ground a general theory of moral uncertainty. I'll set aside other forms of normative uncertainty such as uncertainty about prudence or rationality. I won't introduce new labels. So in this section, when I speak of 'EVM', I don't mean the theory of axiological uncertainty I've so far discussed. I mean the more general idea that under moral uncertainty, you ought to maximise the expectation of a quantity that represents the moral worth, or choiceworthiness, or the amount of moral reasons, assigned to options by moral theories. For this idea to make sense, EVM mustn't range over betterness-relations only. There must be a more general relation between options that all moral theories induce. I'll take 'moral preferability' to be such a relation. So in this section, by ' $T_{1}$ ', ' $T_{2}$ ', ' $T_{3}$ ', ... I'll mean theories about moral preferability. And by ' $a \geq_{i} b$ ', ' $a>_{i} b$ ' and ' $a \sim_{i} b$ ' I'll mean, respectively, that $a$ is morally weakly preferable to, or strictly preferable to, or equally as preferable as $b$, according to $T_{i}{ }^{136}$

The application of EVM to moral theories raises various questions. ${ }^{137}$ In what follows, I'll focus on one issue only: on whether moral theories generally satisfy the axioms of decision theory. If they don't, this raises our two familiar problems for EVM. First, it's unclear how to explicate cardinal intra- and intertheoretic comparisons for such theories-or how to even understand EVM. Second, it's implausible that the m-value facts satisfy these axioms if they range over such theoriesor that EVM is true. These problems (or at least the first one) are sometimes acknowledged. But my sense is that their urgency is underestimated. In response to the question of how to represent moral theories by value functions, Jacob Ross says:

[F]or any theory that tells us what to do in [...] cases of uncertainty and that satisfies certain minimal coherence conditions, we can construct a value function that indicates not just the ordinal values of one's options, but also ratios among the value intervals between them. (2006a, 25; see 2006b, 754 f.)

136 In applying EVM to moral uncertainty, some authors have taken moral theories to imply 'moral choice-worthiness' relations (see MacAskill 2014), or moral 'value' relations (where that is somehow understood more broadly than axiological value; see Sepielli 2010 or Ross 2006b). However, both 'more choice-worthy than' and 'more valuable than' are, as a matter of meaning, transitive. So these interpretations threaten to rule out intransitive theories from the outset.

137 One important problem is supererogation, for which most standard deontological theories allow. According to these theories, there are options $a$ and $b$ such that $a$ is morally preferable to $b$, but it's not the case that you ought to choose $a$. This raises the problem of how moral preferability and the moral 'ought' are weighed under uncertainty. See e. g. Sepielli (2010, ch.6) or Tarsney (2019b) for a discussion. 
This suggests that any 'minimally coherent' theory will be representable by a value function. ${ }^{138}$ But that's anything but clear. To begin with, a theory can only be represented by ' $a$ ' (single) value function if it satisfies Completeness. But incompleteness abounds among deontological theories, just as among axiologies. There are many plausible pluralist views, say, on which what's morally preferable depends on a range of factors-special obligations, rights, impersonal goodness, and so on. On many such views, there aren't determinate facts about how these considerations weigh against each other in cases of conflict. ${ }^{139}$ These theories are incomplete. Now as the theorems from the last section show, EVM doesn't presuppose Completeness, or representability by a single value function. That's one reason why these theorems were crucial to even begin examining how far EVM can cover moral uncertainty. But similar problems arise for Transitivity, Continuity and Independence.

\section{Transitivity}

Start with Transitivity. There are many moral theories that prima facie violate this axiom. Consider a person-affecting view of population ethics according to which, if you have a choice between bringing about two worlds, it's preferable to bring about the world in which the total wellbeing of all the people existing in both worlds is greater, and if this total wellbeing is equal in both worlds, the worlds are equally preferable. ${ }^{140}$ Suppose we have some cardinal concept of wellbeing, and consider the following five worlds-where the first number refers to Therese's and the second number to Philine's level of wellbeing, and ' $\Omega$ ' indicates that the person doesn't exist in that world:

$$
a:(2, \Omega), \quad b:(1,3), \quad c:(\Omega, 2), \quad d:(3,1), \quad \text { and } \quad e:(2, \Omega) .
$$

According to the person-affecting view, $a>b, b>c, c>d$ and $d>e$. Since $a$ and $e$ seem to be the same options, our view is prima facie intransitive. ${ }^{141}$

138 Sepielli (2010, ch. 5) also suggests the same method for cardinalisation. However, he doesn't even mention that a theory has to satisfy certain conditions for this method to be applicable. MacAskill (2014) also suggests the same method, and does mention that moral theories have to satisfy certain axioms for that method to work, but doesn't discuss whether they generally do.

139 Such a pluralist theory, explicitly implying incompleteness, is defended in Nagel (1979). Views that allow for moral dilemmas are also plausibly construed as incomplete. See e.g. Richardson (1994, $115 \mathrm{ff}$.).

140 For a defence of such a view, see e. g. Roberts (2003).

141 I thank John Broome for this example. Note that for simplicity, in this section, I'll consider a transitivity-condition on the non-reflexive relation $>$ rather than the reflexive relation $\succeq$. As is 
The intransitivity arises because according to our view, whether the wellbeing of a person in a world matters depends on whether she exists in the world we compare it with. More generally, the view features 'alternative-dependency': it says that the moral worth of an option depends on its alternative. This easily leads to such intransitivities. And unfortunately, as Tim Willenken (2012) has shown, common sense morality is full of alternative-dependency. Consider, for example, the following common sense principles:

Numbers: If faced with a pairwise choice between saving a lesser number of people from some harm and a greater number of people from that same harm, it's morally preferable to save the greater number.

Dominance: If faced with a pairwise choice between two options $a$ and $b$, where each individual is at least as well off and someone is much better off if you choose $a$ rather than $b$, it's morally preferable to choose $a$.

No Pushing: If faced with a pairwise choice between pushing one person off a bridge to his death in order to block a trolley and letting several other people get killed by that trolley, it's morally preferable to let the greater number get killed.

As Willenken has shown, these three principles generate a prima facie deontic cycle. ${ }^{142}$ And such examples could be multiplied with ease. Common sense morality is full of alternative-dependency, and thus full of prima facie intransitivities.

However, the case isn't as simple. These examples raise the question of how options, or outcomes, should be individuated. Suppose a theory implies that $a>$ $b, b>c$, and $c>a$, on grounds of alternative-dependency. One might say it treats $a$-when- $b$-was-the-alternative $\left(a_{b}\right)$ as different from $a$-when- $c$-was-the-alternative $\left(a_{c}\right)$. After all, it takes the relevant alternatives to matter. For instance, one might say that our person-affecting theory treats $(2, \Omega)_{(3,1)}$ as distinct from $(2, \Omega)_{(1,3)}$. If we bring about $(2, \Omega)$ by rejecting $(3,1)$, we've made Therese worse off than she could have been. But there's no one for whom that's true if we bring about $(2, \Omega)$ by rejecting $(1,3)$. And since our theory is particularly concerned with such harms, this more fine-grained description is a better specification of (what matters in) an outcome for our theory. In individuating outcomes coarsely, we ignore features of the world that according to our view matter morally. Now if we do individuate outcomes according to their alternatives, alternative-dependency no longer grounds

easily verified, the condition on $\geq$ implies that on $>$. Suppose $a>b$ and $b>c$. Then by the transitivity of $\geq, a \geq c$. Moreover, if $c \geq a$ were to hold, by the transitivity of $\geq, c \geq b$ would hold-which contradicts $b>c$. So $c \geq a$ cannot hold, and we have $a>c$.

142 See Willenken $(2012,546)$ for an example. 
intransitivity. The claims $a_{b}>b_{a}, b_{c}>c_{b}$, and $c_{a}>a_{c}$ are perfectly consistent with Transitivity. So there's a general strategy by which we can render theories that seem to violate this axiom consistent with it.

There's a downside to this strategy. Many theories that are complete under a coarse-grained individuation of outcomes will become very incomplete under such a fine-grained one. Take the person-affecting view again. If we individuate outcomes only by the people who exist in them and their level of wellbeing, the theory tells us for any two outcomes which of them is preferable. That isn't so if we individuate outcomes more finely. The theory doesn't imply any ordering of the worlds $(2, \Omega)_{(3,1)}$ and $(1,3)_{(2, \Omega)}$. Therese could have been better off in both $(2, \Omega)_{(3,1)}$ and $(1,3)_{(2, \Omega)}$. But are these worlds equally preferable because the harm is the same (1 unit of wellbeing); or is $(2, \Omega)_{(3,1)}$ preferable because in this world Therese is better off than in $(1,3)_{(2, \Omega)}$ ? The theory is silent on this. It's not defined to order these two outcomes. And there's no reason why it should be. It's logically impossible for you to face a choice between $(2, \Omega)_{(3,1)}$ and $(1,3)_{(2, \Omega)}$. In such a choice, you could either choose $(1,3)$ by rejecting world $(2, \Omega)$, or choose $(2, \Omega)$ by rejecting the altogether different world $(3,1)$. But you can't possibly have these two options. It's an impractical comparison. Individuation of outcomes in terms of their alternatives always leads to impractical comparisons. But deontic moral theories are designed to guide your decision-making. And they can do this perfectly well while being silent on impractical comparisons. So as they stand, they generally won't give advice about such comparisons.

There's an important structural difference here between axiologies and deontic moral theories. An axiology is a claim about which worlds are better than which, or about which properties contribute to the value of worlds. But actual worlds are infinitely fine-grained, or characterised by an infinite number of properties. And for each of these properties, an axiology should say whether it contributes to value or not. An axiology is fragmentary, not fully specified or welldefined, if it's silent on how fine-grained outcomes or prospects are ordered. This isn't to say that an axiology must be 'complete' in my technical sense-that it must imply that any two options are comparable. But it must imply for any two outcomes or prospects that they're equally good, or that one is better, or that they're incomparable. If it's simply silent on the comparison, then to at least one of them it hasn't assigned any (precise or rough) value, which by its nature it should. A fully specified axiology may feature the verdict of an absence (of precise valuecomparisons); but it can't feature the absence of any verdict.

So axiologies must imply a verdict on impractical comparisons. Modal properties, such as what could have been the case instead of a world, are properties of worlds just like the number of people or the amount of welfare they contain. So an axiology is fragmentary if it doesn't specify whether such modal properties 
contribute to value or not. Whether we can face a choice between two worlds is irrelevant from an axiological point of view. Indeed, our framework featured other impractical comparisons, like comparisons between prospects with different underlying probability distributions over axiologies. This wasn't a principled problem. In contrast, deontic moral theories (especially deontological ones ${ }^{143}$ ) aren't in the business of saying which properties contribute to value. They're designed to guide your decision-making. So by their standard, whether we can face a choice between two worlds is pivotal. There's nothing fragmentary or underspecified if a deontic moral theory is silent on impractical comparisons.

What does that mean for present purposes? Fortunately, from the point of view of Completeness and Transitivity, I think this difference doesn't matter much. There are representation theorems that allow for incompleteness, and if the other conditions of these theorems could be satisfied even under a fine-grained individuation of outcomes, these theorems would serve their purpose. If for some two options $a$ and $b$, a deontic theory neither implies that $a \geq b$ nor that $b \geq a$, nothing in the theorems requires that this must be because of an explicit verdict of incomparability. So for the purposes of representation, we could treat the absence of a verdict like the verdict of an absence. We'd blur this distinction on a formal level. But we could bear it in mind, and no great harm would be done. So given that we have theorems allowing for incompleteness, the incompleteness emerging from reindividuation wouldn't in itself present a problem. Let's thus turn to Continuity and Independence.

\section{Continuity}

Questions of continuity are less often discussed in deontological ethics. But as with Transitivity, many standard deontological theories prima facie violate this axiom. That's because, prima facie, many deontological constraints are best captured by probability-thresholds. Consider the violation of rights. According to common sense deontology, you can respect a person's rights even if you take some

143 There's a question about whether deontic consequentialist theories imply verdicts concerning impractical comparisons. One might say they're also just designed to guide your decisionmaking, and since you can't face impractical comparisons, they won't imply any verdict about them either. However, there's a straightforward sense in which a deontic consequentialist theory can be extended or interpreted to imply such verdicts: we can interpret it as saying an option is 'weakly morally preferable' to another if and only if the prospect it represents is at least as good. Interpreted thus, it will inherit all the richness of its underlying axiology. So when I speak of the problems of deontic moral theories, I generally have in mind deontological theories especially. 
risk of killing her for the sake of a minor pleasure-driving past her at sufficient distance on your way to a restaurant, say. But you violate her rights if you take a considerable risk of killing her for that pleasure. Yet whether you violate her rights isn't a matter of degree: you either violate them or you don't. So when does the constraint that you ought to respect people's rights apply? It seems congenial to deontology to accept a probability threshold. ${ }^{144}$ But such thresholds lead to incontinuity. Suppose you violate someone's rights if you take more than a $1 \%$ risk of killing her for the sake of a minor pleasure. Suppose there's thus a constraint against taking such a risk, but not against taking a risk of $1 \%$ or less. And suppose not violating anyone's rights is always preferable to violating someone's, and that if you don't violate anyone's rights, risking fewer deaths is preferable to risking more. Now consider

$f \quad$ killing Juliette with $100 \%$ probability;

$g \quad$ killing Lenardo and Valerine with $1 \%$ probability; and

$h \quad$ killing Juliette with $1 \%$ probability.

Our view implies that $h>g$ and $g>f$. But there's no probability $p \in] 0,1[$ such that $p f+(1-p) h>g$. For any $p>0, p f+(1-p) h$ involves a risk of more than $1 \%$ of killing Juliette, and thus violates her rights. So $g$ will be preferable to it.

Again, it's easy to find other examples where such thresholds seem congenial to deontology. Consider whether it's permissible to kill one person to save ten others. On common sense deontology, this depends on whether that person forfeited her right not to be killed-by intending to kill the ten, say. Yet you don't need absolute certainty that she forfeited her right, or else the permission would be irrelevant in practice. You only need it to be clear 'beyond reasonable doubt'. What does that mean? A natural interpretation is again that there's a threshold, such that you can permissibly kill someone for the sake of saving ten others if and only if the probability of her innocence isn't more than this threshold. Suppose this threshold is $3 \%$. And suppose not killing anyone impermissibly is always preferable to killing someone impermissibly, and that permissibly killing fewer people is preferable to permissibly killing more. Consider

i killing William, where William has a $10 \%$ probability of being innocent;

j killing Margaret and Clara, where Margaret and Clara both have a 3\% probability of being innocent; and

$k \quad$ killing William, where William has a 3\% probability of being innocent.

144 For further reflections on deontological approaches to uncertainty, see e. g. Jackson and Smith (2006), Tenenbaum (2017), Lazar (2018) or Tarsney (2018b). 
Our view says that $k>j$ and $j>i$. But there's no probability $p \in] 0,1$ [ such that $p i+(1-p) k>j$. For any $p>0, p i+(1-p) k$ involves killing someone who has a probability of more than $3 \%$ of being innocent, and is thus impermissible. So $j$ will be preferable to it. ${ }^{145}$

More examples could easily be given-concerning cases where it's unclear whether you have a promissory obligation to someone, are using them as a mere means, are violating their autonomy, and so on. Prima facie, many standard deontological theories violate Continuity. Is there a strategy to resist this? Reindividuating outcomes might again be natural. Consider our first example. Option $f$ was to kill Juliette with certainty, option $h$ to kill her with a $1 \%$ chance. Suppose you chose $h$, and it resulted in Juliette's death. For our theory there will have been a categorical difference between your action and the action of choosing $f$ and Juliette's certain demise. You didn't violate her rights. So we might say our theory treats the resulting deaths as distinct-implying that Juliette is suffering mere injury as an outcome of $h$, but injury and insult (i. e. rights-violation) after $f$. More generally, probability threshold-views seem to posit a categorical difference between an outcome that had more and one that had less than this probability of coming about, however similar they otherwise are.

Yet, natural as such a reindividuation may be, it doesn't help make our deontic theories continuous. Once we've distinguished the outcome 'Juliette is dead, and suffered the insult of a rights-violation' (of $f$ ) from 'Juliette is dead, but suffered no insult' (of $h$ ), we'll again face impracticalities. Consider the option ' $p f+(1-$ $p) h$ ', with $p \in] 0,1$ [. One possible outcome of this option is that Juliette suffered an insult, and another is that she suffered none. But on common sense views of rights, rights violations are determined ex ante, rather than ex post through brute moral luck. So you can't face such a prospect in practice. Whatever you do, you'll either violate her rights or you won't, for all possible outcomes of your choice. So you can't face a prospect like ' $p f+(1-p) h$ '. This option not only contributes to impractical comparisons with others, as in the cases we've encountered above. It's an impractical option, all in itself. Deontic theories (especially deontological ones) generally won't imply verdicts concerning such options. There's no reason why they should. So they generally won't imply any verdicts of the form ' $p f+(1-p) h>$ $g$ ', or ' $g>p f+(1-p) h$ '. But such verdicts are precisely what Continuity would require. So reindividuating outcomes won't help with this axiom.

The difference between Continuity and Transitivity is this. In either case, the pertinent reindividuation will lead to impracticalities, and thus to widespread

145 This example is taken from Jackson and Smith (2006); the threshold view is accepted by Aboodi et al. (2008). 
incompleteness. This is a problem with Continuity. Continuity features what we might call a 'conditional minimal completeness constraint'. It requires that, if a theory implies that $a>b$ and $b>c$, it must also make some third judgment involving $p a+(1-p) c$ (viz., that $p a+(1-p) c>b$ for some $p \in] 0,1[$ ) - or can't be fully incomplete with respect to this latter option. Now even under a fine-grained individuation of outcomes via their probabilities, there'll be many (standard, practical) options for which deontic moral theories imply the first two judgments, and thus satisfy the antecedent of the conditional completeness constraint. So then Continuity will require at least minimal completeness with respect to $p a+(1-p) c$, which the theories will fail to deliver. Transitivity also features a conditional minimal completeness constraint. It requires that if a theory implies that $a>b$ and $b>c$, it must also make some third judgment involving $a$ and $c$ (viz., that $a>c$ ). However, under a fine-grained individuation of outcomes via their alternatives, there won't be any options for which our theories imply the first two judgments, and thus satisfy the antecedent of the conditional constraint. They might imply that $a_{b}>b_{a}$ and $b_{c}>c_{b}$. But this doesn't force them to any third judgment. They would be so forced if they implied that $a_{b}>b_{a}$ and $b_{a}>c_{b}$. But this they won't imply. So even if theories fail to deliver judgments like $a_{b}>c_{b}$, that's no problem, since Transitivity won't require them to.

Again, I take it, there's an important difference here between axiologies and deontic moral theories. Axiologies aren't designed to guide your decision-making. So there doesn't seem to be a principled reason why they shouldn't imply verdicts concerning impractical options, or deliver the minimal completeness that Continuity requires. Our framework featured other impractical options, like prospects in which it depends on the correct axiology whether you'll kill a non-human animal. So as indicated in Section 3.2, I take it the strategy of reindividuating outcomes can more successfully be applied in the case of axiologies-e. g., axiologies that treat rights infringements as a bad thing. It can help us render axiologies that prima facie violate Continuity consistent with it. It just cannot help with deontic views.

Is there another strategy to resist prima facie violations of Continuity? One might think there is. Intuitively, discontinuities arise through thresholds, or sudden leaps in the graph that designates our options' worth. So perhaps we could assume that the relevant deontological worth-functions have continuous drops instead of discontinuous leaps. Rather than saying that a risk of $1 \%$ marks a cutoff point for the violation of rights, say, we could say it marks a point where our options' worth decreases drastically but continuously-as in the following illustration: 

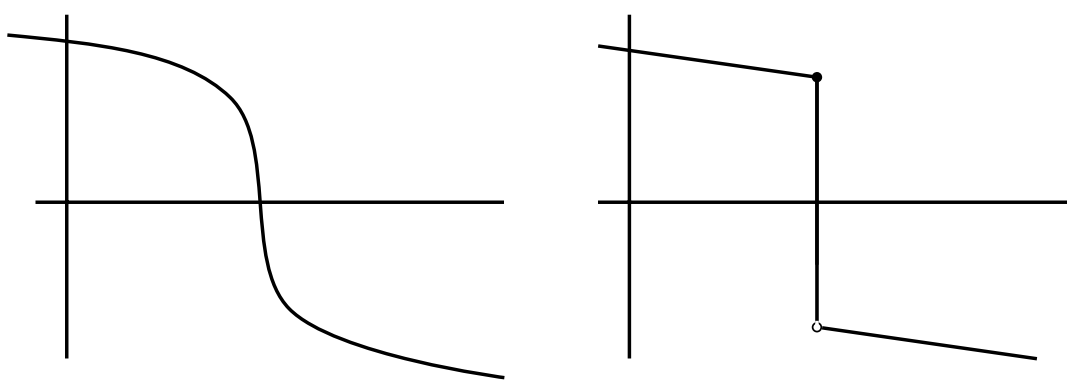

Fig. 6.1: Graphs to illustrate continuous drop (left) versus discontinuous leap (right).

Indeed, one might think that's a more charitable interpretation of deontological views, as thresholds seem mysterious or arbitrary.

But this strategy isn't fully satisfying either, at least with standard deontological theories like the ones we've been considering. Such views operate with all-or-nothing concepts. There's an important qualitative difference in whether or not you violate rights, say. Your actions are generally permissible as long as you don't, and impermissible as soon as you do. And you can't violate rights a little bit. Of course, not all rights violations are equally grievous, as there are more and less weighty rights. But even the less important rights will either be violated or not, and that will be a qualitative difference. All-or-nothing concepts are at the heart of deontology, and in the face of uncertainty, it seems they'll have to be captured by thresholds. If we reinterpret such views as continuous, we may produce extensionally similar analogues of them. And working with these analogues may be the best we can do. But it doesn't present a fully satisfying theoretical solution to the problem of discontinuous theories. Moreover, as we'll presently see, even if we render our views continuous, this won't help make them satisfy Independence. So even if we accept this strategy in the face of Continuity, it won't solve the general problem we're concerned with.

There's a final strategy one could propose. It's also a possible strategy concerning Independence. So let me discuss it in that context, and turn to this final axiom now.

\section{Independence}

If what I've said is correct, the case of Independence is even more problematic. As with the other axioms, many standard moral theories prima facie violate it. Consider fairness. According to a common sense idea, a distribution of a good is fair if and only if people's claims to this good are satisfied in proportion to their 
strength. If a good can't be divided, it's fairest to put on a lottery, in which people's chances of receiving this good are proportional to the strength of their claims. ${ }^{146}$ And a distribution or lottery is less fair the further away it is from (expected) proportionate satisfaction of claims. Now suppose Care and Helen both have a claim to some indivisible good $G$, but Care's is twice as strong as Helen's. Consider the options

$l$ Care gets $G$; and

$m$ Helen gets $G$.

On the present theory, $l>m$, since $l$ comes closer to a distribution in which their claims are satisfied in proportion to their strength. However, suppose you have a biased coin that lands tails $2 / 3$ of the time, and you can choose among the lotteries

$n \quad$ Care gets $G$ no matter how the coin lands; and

$o$ Helen gets $G$ if the coin lands heads, and Care if it lands tails.

Given that $G$ is indivisible, $o$ is the fairest option available. So we have $o>n$. However, since $n$ seems equivalent to $1 / 3 l+2 / 3 l$, and $o$ equivalent to $1 / 3 m+2 / 3 l$, this theory seems to violate Independence. ${ }^{147}$

Again, it's clear why it does. According to this theory, what matters about an outcome is not only what actually happens in it, but also what could have happened, and with what probability, instead of it. So we can't evaluate the outcomes of our actions independently of one another. For the same reason, the above view of rights will also violate Independence. On this view, again, you violate someone's rights if you take a risk of more than $1 \%$ of killing her for the sake of a minor pleasure, but not if the risk is $1 \%$ or less. Consider our options

$f \quad$ killing Juliette with $100 \%$ probability;

$g$ killing Lenardo and Valerine with $1 \%$ probability; and

$h$ killing Juliette with $1 \%$ probability.

The theory implies that $h>g$. But for $p=0.99$, it implies that $p g+(1-p) f>$ $p h+(1-p) f-$ since the latter but not the former option violates someone's rights. And something similar will hold for many related views. Prima facie, violations of Independence are again very common in standard deontology.

Unfortunately, however, neither of the two strategies we encountered with Transitivity and Continuity works with Independence. First, the problem with reindividuating outcomes via the probability with which they came about is

146 For a defence of such a view, see e. g. Broome (1990).

147 See Diamond (1967) for a similar example. 
exactly the same as with Continuity. Independence also features a conditional minimal completeness constraint. It requires that, if a theory implies that $a>b$, it must also make verdicts involving some compound option $p a+(1-p) c$ (viz., that $p a+(1-p) c>p b+(1-p) c$ for all $c)$. And even under a fine-grained individuation of outcomes via their probabilities, there'll be many standard, practical options for which deontic moral theories imply the first judgment, and thus satisfy the antecedent of the conditional completeness constraint. So then Independence will require minimal completeness with respect to $p a+(1-p) c$. But this will be an impractical option. This won't be a problem for axiologies. So as indicated in Section 3.2, I take it the strategy of reindividuating outcomes can help us render axiologies that prima facie violate Independence consistent with it. But theories designed to guide action will remain silent on such impractical options, and won't deliver the relevant minimal completeness.

Second, violations of Independence don't depend on probability-thresholds. Even if we interpret our theories in terms of continuous drops rather than discontinuous leaps, it remains true that what matters according to them is not only what actually happened, but also what could have happened instead. This very general fact causes violations of Independence, and we don't avoid it by making our theories continuous. Consider our view of rights-violations again. Suppose the moral worth of options decreases drastically but continuously when we take a risk of more than $1 \%$ of killing someone. Then adding an additional risk of killing Juliette will decrease the worth of 'killing Lenardo and Valerine with $1 \%$ probability' less than it will decrease the worth of 'killing Juliette with $1 \%$ probability'. And this will lead to violations of Independence. So even if we grant that this strategy allows us to satisfy Continuity, it doesn't help with Independence. Whatever we think about interpreting deontological views as continuous, doing so doesn't help with the general problem we're concerned with.

As indicated, there's a final move in response to violations of Independence, which could also be made with respect to Continuity. It builds on reindividuating outcomes. I've shown that reindividuating outcomes will render our theories too incomplete even to satisfy the axioms of Continuity and Independence. This is because reindividuating outcomes will produce impractical options, and our theories aren't designed to order such options. So what we could do is this. We could extend our theories and make them imply verdicts on impractical options; and we could do that in ways that accord with the relevant Continuity and Independence constraints. As far as I see, that would be possible. And as first-order judgments of moral preferability go, it wouldn't alter these views in any practically relevant manner. We'd keep all their verdicts on practically possible options and comparisons, and only extend them with respect to options and comparisons you can't 
face in practice. So one might argue that such extensions would be innocuous, and not distort our views in any problematic respect.

However, there are problems with this move too. For one thing, by so extending our views, we'd turn them into different theories-indeed, theories of a different kind. Again, deontic moral theories have a specific nature, and that's fundamentally different from that of axiologies. From an axiological point of view, it's irrelevant whether options can figure in practical choice. So there's something fragmentary about an axiology that implies no verdict on impractical options. But deontic moral theories are just designed to guide choice. By a deontic standard, there's nothing fragmentary about silence on impracticalities. In fact, at least with the deontological theories we've been considering, there will often be no basis within these views on which they could imply verdicts on impractical comparisons or options. Hence by making our moral theories order impractical options, we wouldn't somehow finish them, complement them where otherwise they'd be fragmentary. We'd change their very nature. We'd basically turn them into axiologies, without any basis for doing so implied by the theories themselves.

Moreover, even though our extensions would have no practical implications as far as first-order judgments of moral preferability are concerned, they would have practical implications under uncertainty. Consider again the options of killing Juliette with $100 \%$ probability $(f)$, killing Lenardo and Valerine with $1 \%$ probability $(g)$, and killing Juliette with $1 \%$ probability $(h)$. And suppose we reindividuate outcomes and supplement our theories with verdicts such that, for some $p$ and $q \in] 0,1[, p f+(1-p) h>g$ and $g>q f+(1-q) h$. The precise verdicts will determine where on the value scale between $f$ and $h$ the value of $g$ lies. And that in turn will determine how important it is to choose $g$ rather than $f$, according to our theory. And while this may be irrelevant if we're certain of this theory and face a choice between $f$ and $g$, it's not irrelevant if we have to weigh it against a theory according to which $f$ is morally preferable to $g$. So there'd be no basis within the deontological views to make these extensions, and yet they'd have significant implications under uncertainty. Thus even if these extensions are the best we can work with in practice, they don't present a fully convincing theoretical solution to our problem. In providing a theory of uncertainty about these extended cousins of our deontological views, we're not giving a theory of uncertainty about these deontological views.

\section{Conclusion}

In conclusion, a great number of standard deontic views don't satisfy our axioms, and there's no altogether convincing way in which we can interpret them as do- 
ing so. This casts doubt on whether the argument I've been developing can readily ground a general theory of moral uncertainty. A fortiori, it casts doubt on whether our argument can readily ground an even more general theory of normative uncertainty. Also, I've now addressed only one problem with understanding EVM as a general theory of moral or normative uncertainty. There remain other problemsfor instance, the question of how to accommodate supererogation. ${ }^{148}$ Even disregarding the question I've discussed, these other problems would still need to be solved.

Let me turn back to axiologies again. I've considered whether the strategy of reindividuating outcomes can render theories that prima facie violate Continuity and Independence consistent with these axioms. I've pointed out a problem with this strategy when applied to deontic views: once we individuate outcomes finely, many standard deontic theories will be too incomplete to satisfy Continuity and Independence, since they're designed to guide your decision-making. But I've suggested this isn't a problem when we apply the strategy to axiologies: axiologies aren't designed to guide your decision-making. Does this mean we can always apply this strategy to axiologies? In other words, does it mean we can see all possible axiologies as satisfying Independence and Continuity, provided we individuate outcomes properly?

It doesn't. Importantly, the strategy only works for axiologies on which the relevant fine-grained features of outcomes-such as facts about what could have happened, or about the probability with which something happened-are indeed good- or bad-making features of outcomes. ${ }^{149}$ I take it that on the most natural axiological versions of the deontic views we've considered, this is the case. For instance, I take it the most natural rights- or fairness-sensitive axiologies say that the fact that someone's rights have been violated (e. g., that Juliette had more than a $1 \%$ chance of being killed) or that some state of affairs came about unfairly (e. g., that Helen had a disproportionately low chance of getting some good) are badmaking features of outcomes. They say that how good some state of affairs is depends among else on its modal properties. So they say the relevant fine-grained features of outcomes do indeed matter axiologically.

In principle, however, this needn't be. There are axiologies that violate Continuity and Independence, but not because they consider such features as goodor bad-making properties of outcomes. Consider Continuity. Take an axiology on which the value of wellbeing lexically dominates that of beauty-in that a prospect is better than another whenever it expectably leads to more wellbeing, but ceteris

148 See footnote 137.

149 For similar considerations, see e. g. Broome (1991, 103 f.). 
paribus better if it expectably leads to more beauty. This welfare/beauty-theory doesn't satisfy Continuity. But the reason for this isn't that on this axiology, some extra modal feature of an outcome makes an axiological difference. The theory doesn't, for example, treat an instance of beauty as more or less valuable depending on whether some wellbeing could have materialised instead of it. It just treats wellbeing as lexically better. Or at least, that's the most natural version or interpretation of this view. So as far as I see, the strategy of reindividuating outcomes couldn't successfully be applied to it. Or consider Independence. Take an axiology on which fairness is purely a consideration at the level of prospects. On this axiology, ceteris paribus, one prospect is better than another if on that prospect people's chances of receiving some good are proportional to the strength of their claims. But an unfair causal history isn't a bad-making feature of an outcome. So on this axiology, ex ante it's best to give people fair chances. But ex post the chances that people have had are irrelevant, and it's only the actual distribution of goods that matters. This too is a possible theory. And as far as I see, the strategy of reindividuating outcomes couldn't successfully be applied to it. So I think we can't understand all possible axiologies as satisfying Continuity and Independence. Or again, our argument cannot ground a fully general theory of axiological uncertainty. It can only ground a theory of uncertainty about those axiologies that are, or can successfully be seen as being, $\mathrm{N}^{\star}$-conformable.

However, in contrast to the case of deontic view, my sense is that most standard axiologies satisfy our conditions, and that the axiologies we ultimately strictly exclude are comparatively rare and implausible. Take the two axiologies I've just mentioned. Presumably, we should have some nonzero credence in them. But they certainly don't seem very plausible. On the contrary, the extreme kind of lexical priority contended by the former, and the distinction between ex ante and ex post considerations assumed by the latter, make them rather dubious. More generally, at the level of axiologies, Continuity and Independence do seem to express compelling thoughts. Thus while its restrictions are non-trivial, I think our theory is still an interestingly general theory of axiological uncertainty. That's part of why, in this book, I've focused on the narrower problem of axiological, rather than the general problem of moral or normative uncertainty.

\subsection{Conclusion}

In this book, I've explored an axiomatic approach to the problem of axiological uncertainty, and to the idea of Expected Value Maximisation in particular. I first introduced a basic representation theorem (Chapter 2), and argued that-modulo 
the fundamental metaphysical problem of intertheoretic comparisons, the presupposition of axiological probabilities as primitives, and the existence of intraand intertheoretic incommensurabilities-this theorem can help answer the question of meaning and the question of truth about EVM (Chapter 3). I then addressed these restrictions one by one. I argued that epistemic norms can provide a constructivist ground for intertheoretic comparisons (Chapter 4). I furnished the overall theory with an account of axiological credences, and thus paved the way for applying it in real life (Chapter 5). And I extended the theorem to cover axiologies that involve intra- or give rise to intertheoretic incommensurabilities (Chapter 6).

Naturally, there remain many problems for further research. On the one hand, there are further philosophical questions. For instance, in discussing the axioms, I've often restricted myself to comparing their plausibility in our context with their status in other contexts. To make a more complete case for EVM, it would be necessary to say more in their defence. Similarly, it would be interesting to explore constructivism about intertheoretic comparisons in more depth, and spell out some plausible actual implications of it. And most urgently perhaps, this last chapter raised problems for the theory of moral uncertainty, and it would be important to make progress on these. On the other hand, there remain many open technical problems. It may be worthwhile to seek theorems for incomplete orderings that yield unique separations of subjective probabilities and state-dependent utilities and don't rely on the strong Reduction Axiom. It ultimately seems important to explore results for state-dependent utilities that allow for incomparability in values as well as fuzzy credences. And since there are infinitely many axiologies, it would certainly be desirable to extend the results of this book to a framework that allows for an infinite state-space.

As usual in moral philosophy, I can't be certain that my arguments are sound. But I hope this book has at least shed a new light on some difficulties in the theory of normative or evaluative uncertainty and on a possible path to a solution. In particular, I hope I've shown that Expected Value Maximisation is neither selfexplanatorily clear nor trivially true, but that the axiomatic approach provides a promising strategy to explicate and ground it. And I hope this has some value. 
